\title{
Topology of families of affine plane curves
}

\author{
by Hà Huy Vui (Hanoi) and Pham Tien Son (Dalat)
}

\begin{abstract}
We determine bifurcation sets of families of affine curves and study the topology of such families.

1. Introduction. Let $f_{\alpha}(x, y)$ be a family of polynomials of two complex variables $(x, y) \in \mathbb{C}^{2}$ whose coefficients are polynomial functions of $\alpha \in \mathbb{C}^{n}$. We consider the family of affine curves $\left\{(x, y) \in \mathbb{C}^{2} \mid f_{\alpha}(x, y)=0\right\}$.

In this paper, we first determine the bifurcation set $B_{f}$, i.e., the smallest set of parameters $\alpha$ such that the family is equisingular outside this set. Then applying this result, we introduce the notions of semi-cycles vanishing at infinity and study the topology of the family. Finally, we show that our results imply some well-known facts on the topology of polynomial functions $([2],[3],[6],[7])$.
\end{abstract}

2. Bifurcation set of families of affine plane curves. Let $f_{\alpha}(x, y):=$ $P(x, y, \alpha), \alpha \in \mathbb{C}^{n}$, be a family of polynomials of two variables whose coefficients are polynomials of $\alpha$.

2.1. Definition. The family of affine curves $\left\{(x, y) \in \mathbb{C}^{2} \mid f_{\alpha}(x, y)=0\right\}$, $\alpha \in \mathbb{C}^{n}$, is said to be equisingular outside a set $B \subset \mathbb{C}^{n}$ if for all $\alpha^{0} \notin B$ there exist a neighborhood $U_{\alpha^{0}}$ of $\alpha^{0}$ and a diffeomorphism $h$ such that the diagram

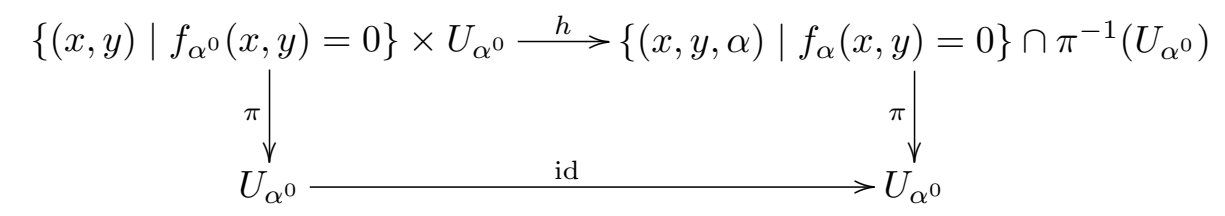

is commutative, where $\pi$ is the second projection.

1991 Mathematics Subject Classification: 32S55, 57M25, 57Q45.

Key words and phrases: affine curves, singularity, equisingularity, Milnor number.

Supported in part by the National Basic Research Program in Natural Sciences, Vietnam. The second author would like to thank the AUBONNE for financial support. 
Let $B_{f}$ be the smallest set of parameters $\alpha$ such that the family $\left\{f_{\alpha}(x, y)=0\right\}$ is equisingular ouside $B_{f}$. We call $B_{f}$ the bifurcation set of the family.

2.2. The following assumptions will be needed throughout the paper:

- $\operatorname{deg}\left(f_{\alpha}\right)=\operatorname{deg}_{y}\left(f_{\alpha}\right)=d=$ const;

- the curves $\left\{f_{\alpha}(x, y)=0\right\}$ are all reduced.

Let $l$ be the linear function defined by $l(x, y)=x$. By assumption, the restriction map

$$
l_{\alpha}: f_{\alpha}^{-1}(0) \rightarrow \mathbb{C}, \quad(x, y) \mapsto x,
$$

is proper for each $\alpha \in \mathbb{C}^{n}$.

Let $\delta(x, \alpha):=\operatorname{disc}_{y}\left(f_{\alpha}(x, y)\right)$ be the discriminant of $f_{\alpha}$ with respect to $y$. Then we may write

$$
\delta(x, \alpha)=q_{k}(\alpha) x^{k}+q_{k-1}(\alpha) x^{k-1}+\ldots
$$

where $q_{i}(\alpha), i=0, \ldots, k$, are polynomials of $\alpha$. Put

$$
B_{\infty}:=\left\{\alpha \mid q_{k}(\alpha)=0\right\} .
$$

Denote by $C_{f}\left(f_{\alpha}\right)$ the set of critical values of $f_{\alpha}$.

2.3. Theorem. Assume that $0 \notin C_{f}\left(f_{\alpha}\right)$ for generic $\alpha$. Then the bifurcation set of the family of affine curves $\left\{f_{\alpha}(x, y)=0\right\}$ is precisely the set

$$
B=\left\{\alpha \mid 0 \in C_{f}\left(f_{\alpha}\right)\right\} \cup B_{\infty} .
$$

Proof. We first prove that the family of affine curves $\left\{f_{\alpha}(x, y)=0\right\}$ is equisingular outside $B$. For each polynomial $f_{\alpha}$, define

$$
\operatorname{grad} f_{\alpha}:=\left(\overline{\partial f_{\alpha} / \partial x, \partial f_{\alpha} / \partial y}\right) \text {. }
$$

Assume that $\alpha^{0} \notin B$. Let $U_{\alpha^{0}}:=\left\{\alpha \in \mathbb{C}^{n} \mid\left\|\alpha-\alpha^{0}\right\|<\delta\right\}$ so that $\mathbb{C}^{n} \backslash B$ contains the closure of $U_{\alpha^{0}}$.

By the definition of $B, q_{k}(\alpha) \neq 0$ for all $\alpha \in U_{\alpha^{0}}$; hence there exists $c_{0}>0$ such that if $\alpha \in U_{\alpha^{0}}$, then $\delta(x, \alpha) \neq 0$ on the set $\left\{x \in \mathbb{C}|| x \mid \geq c_{0}\right\}$. By the properties of resultants, the system of equations

$$
\left\{\begin{array}{l}
f_{\alpha}(x, y)=0, \\
\partial f_{\alpha} / \partial y=0,
\end{array}\right.
$$

has no solution on the set $\left\{(x, y) \in \mathbb{C}^{2}|| x \mid \geq c_{0}\right\}$ for any fixed $\alpha \in U_{\alpha^{0}}$. Thus

$$
\partial f_{\alpha} / \partial y \neq 0 \quad \text { for all }(x, y, \alpha) \in V_{\alpha^{0}} \cup \Omega \text {, }
$$


where

$$
V_{\alpha^{0}}:=\left\{(x, y, \alpha) \in \mathbb{C}^{2} \times \mathbb{C}^{n}\left|f_{\alpha}(x, y)=0, \alpha \in U_{\alpha^{0}},\right| x \mid \geq c_{0}\right\}
$$

and $\Omega$ is an open neighborhood of the set

$$
\left\{(x, y, \alpha) \in \mathbb{C}^{2} \times \mathbb{C}^{n}\left|f_{\alpha}(x, y)=0, \alpha \in U_{\alpha^{0}},\right| x \mid=c_{0}\right\} .
$$

On the other hand, by the definition of $U_{\alpha^{0}}$, one has

(2) $\operatorname{grad} f_{\alpha} \neq 0$ for all $(x, y, \alpha) \in \mathbb{C}^{2} \times U_{\alpha^{0}}$ with $f_{\alpha}(x, y)=0,|x| \leq c_{0}$.

From (1) and (2) we conclude that there exist smooth vector fields

$$
\begin{aligned}
\xi^{j}(x, y, \alpha) & =\left(\xi_{1}^{j}(x, y, \alpha), \xi_{2}^{j}(x, y, \alpha)\right), \\
\eta^{j}(x, y, \alpha) & =\left(\eta_{1}^{j}(x, y, \alpha), \eta_{2}^{j}(x, y, \alpha)\right), \quad j=1, \ldots, n,
\end{aligned}
$$

such that

$$
\left\{\begin{array}{l}
\left\langle\xi^{j}(x, y, \alpha), \operatorname{grad} f_{\alpha}(x, y)\right\rangle+\frac{\partial f_{\alpha}}{\partial \alpha_{j}}(x, y)=0, \\
\left\langle\eta^{j}(x, y, \alpha), \operatorname{grad} f_{\alpha}(x, y)\right\rangle+\sqrt{-1} \frac{\partial f_{\alpha}}{\partial \alpha_{j}}(x, y)=0
\end{array}\right.
$$

on the set $X:=\left\{(x, y, \alpha) \in \mathbb{C}^{2} \times \mathbb{C}^{n} \mid f_{\alpha}(x, y)=0, \alpha \in U_{\alpha^{0}}\right\}$, and

$$
\left\{\begin{array}{l}
\xi_{1}^{j}(x, y, \alpha)=0, \\
\eta_{1}^{j}(x, y, \alpha)=0,
\end{array}\right.
$$

for all $(x, y, \alpha) \in V_{\alpha^{0}} \cup \Omega$. (We can construct such vector fields locally and then extend them over $X$ by a smooth partition of unity.)

To shorten notation, we write $e^{j}=(0, \ldots, 0,1,0, \ldots, 0)$ with 1 in the $j$ th place.

Let $\varphi^{j}\left(x^{j}, y^{j}, \alpha^{j}, \tau\right):=\left(\varphi_{1}^{j}\left(x^{j}, y^{j}, \alpha^{j}, \tau\right), \varphi_{2}^{j}\left(x^{j}, y^{j}, \alpha^{j}, \tau\right), \alpha\left(x^{j}, y^{j}, \alpha^{j}, \tau\right)\right)$, $j=1, \ldots, n$, be solutions of the system

$$
\left\{\begin{array}{l}
\frac{d x(\tau)}{d \tau}=\xi_{1}^{j}(x(\tau), y(\tau), \alpha(\tau)), \\
\frac{d y(\tau)}{d \tau}=\xi_{2}^{j}(x(\tau), y(\tau), \alpha(\tau)), \\
\frac{d \alpha(\tau)}{d \tau}=e^{j}, \\
x(0)=x^{j}, \quad y(0)=y^{j}, \quad \alpha(0)=\alpha^{j},
\end{array}\right.
$$

and $\psi^{j}\left(u^{j}, v^{j}, \beta^{j}, \tau\right):=\left(\psi_{1}^{j}\left(u^{j}, v^{j}, \beta^{j}, \tau\right), \psi_{2}^{j}\left(u^{j}, v^{j}, \beta^{j}, \tau\right), \beta\left(u^{j}, v^{j}, \beta^{j}, \tau\right)\right)$, $j=1, \ldots, n$, be solutions of the system 
(6)

$$
\left\{\begin{array}{l}
\frac{d u(\tau)}{d \tau}=\eta_{1}^{j}(u(\tau), v(\tau), \beta(\tau)), \\
\frac{d v(\tau)}{d \tau}=\eta_{2}^{j}(u(\tau), v(\tau), \beta(\tau)), \\
\frac{d \beta(\tau)}{d \tau}=\sqrt{-1} e^{j}, \\
u(0)=u^{j}, \quad v(0)=v^{j}, \quad \beta(0)=\beta^{j},
\end{array}\right.
$$

where $\left(x^{j}, y^{j}, \alpha^{j}\right),\left(u^{j}, v^{j}, \beta^{j}\right) \in X$. We conclude from (3) and (5) that

$$
\begin{aligned}
\frac{d}{d \tau} P\left(\varphi^{j}\left(x^{j}, y^{j}, \alpha^{j}, \tau\right)\right) & =\frac{\partial P}{\partial x} \frac{\partial \varphi_{1}^{j}}{\partial \tau}+\frac{\partial P}{\partial y} \frac{\partial \varphi_{2}^{j}}{\partial \tau}+\sum_{k=1}^{n} \frac{\partial P}{\partial \alpha_{k}} \frac{\partial \alpha_{k}}{\partial \tau} \\
& =\frac{\partial P}{\partial x} \frac{\partial \varphi_{1}^{j}}{\partial \tau}+\frac{\partial P}{\partial y} \frac{\partial \varphi_{2}^{j}}{\partial \tau}+\frac{\partial P}{\partial \alpha_{j}} \frac{\partial \alpha_{j}}{\partial \tau}=0,
\end{aligned}
$$

hence that

$$
P\left(\varphi_{1}^{j}\left(x^{j}, y^{j}, \alpha^{j}, \tau\right), \varphi_{2}^{j}\left(x^{j}, y^{j}, \alpha^{j}, \tau\right), e^{j} \tau+\alpha^{j}\right)=0 .
$$

Further, it follows from (4) and (5) that

$$
\begin{aligned}
\frac{d}{d \tau}\left|\varphi_{1}^{j}\left(x^{j}, y^{j}, \alpha^{j}, \tau\right)\right|^{2}= & \frac{d}{d \tau}\left\langle\varphi_{1}^{j}\left(x^{j}, y^{j}, \alpha^{j}, \tau\right), \varphi_{1}^{j}\left(x^{j}, y^{j}, \alpha^{j}, \tau\right)\right\rangle \\
= & \left\langle\frac{d \varphi_{1}^{j}\left(x^{j}, y^{j}, \alpha^{j}, \tau\right)}{d \tau}, \varphi_{1}^{j}\left(x^{j}, y^{j}, \alpha^{j}, \tau\right)\right\rangle \\
& +\left\langle\varphi_{1}^{j}\left(x^{j}, y^{j}, \alpha^{j}, \tau\right), \frac{d \varphi_{1}^{j}\left(x^{j}, y^{j}, \alpha^{j}, \tau\right)}{d \tau}\right\rangle \\
= & 0,
\end{aligned}
$$

hence

$$
\left|\varphi_{1}^{j}\left(x^{j}, y^{j}, \alpha^{j}, \tau\right)\right|=\text { const } \quad \text { for each }\left(x^{j}, y^{j}, \alpha^{j}\right) \in V_{\alpha^{0}} .
$$

Analogously,

$$
P\left(\psi_{1}^{j}\left(u^{j}, v^{j}, \beta^{j}, \tau\right), \psi_{2}^{j}\left(u^{j}, v^{j}, \beta^{j}, \tau\right), \sqrt{-1} e^{j} \tau+\beta^{j}\right)=0 ;
$$

moreover,

$$
\left|\psi_{1}^{j}\left(u^{j}, v^{j}, \beta^{j}, \tau\right)\right|=\mathrm{const} \quad \text { for each }\left(u^{j}, v^{j}, \beta^{j}\right) \in V_{\alpha^{0}} .
$$

Since $\operatorname{deg}\left(f_{\alpha}\right)=\operatorname{deg}_{y}\left(f_{\alpha}\right)=d=$ const, the restriction map

$$
l_{U_{\alpha^{0}}}:\left\{(x, y) \mid f_{\alpha}(x, y)=0, \alpha \in U_{\alpha^{0}}\right\} \rightarrow \mathbb{C}, \quad(x, y) \mapsto x,
$$

is proper. In addition, it is clear from (7) and (8) that the solutions $\varphi^{j}\left(x^{j}, y^{j}, \alpha^{j}, \tau\right)$ and $\psi^{j}\left(u^{j}, v^{j}, \beta^{j}, \tau\right), j=1, \ldots, n$, can be extended over their maximal intervals. 
Now define

$$
h:\left\{(x, y) \mid f_{\alpha^{0}}(x, y)=0\right\} \times U_{\alpha^{0}} \rightarrow\left\{(x, y, \alpha) \mid f_{\alpha}(x, y)=0\right\} \cap \pi^{-1}\left(U_{\alpha^{0}}\right)
$$

by

$h(x, y, \alpha)=\psi^{n}\left(\varphi^{n}\left(\ldots\left(\psi^{1}\left(\varphi^{1}\left(x, y, \alpha^{0}, \operatorname{Re} \alpha_{1}-\operatorname{Re} \alpha_{1}^{0}\right), \operatorname{Im} \alpha_{1}-\operatorname{Im} \alpha_{1}^{0}\right), \ldots\right.\right.\right.$,

$$
\left.\left.\operatorname{Re} \alpha_{n}-\operatorname{Re} \alpha_{n}^{0}\right), \operatorname{Im} \alpha_{n}-\operatorname{Im} \alpha_{n}^{0}\right)
$$

for $(x, y) \in f_{\alpha^{0}}^{-1}(0)$ and $\alpha \in U_{\alpha^{0}}$. We can easily check that the map $h$ is a diffeomorphism, and that $\pi \circ h(x, y, \alpha)=\pi(x, y, \alpha)=\alpha$. This gives a trivialization of the fibration over the set $U_{\alpha^{0}}$.

We next prove that the set $B$ is smallest. By contradiction, assume that for some $\alpha^{0} \in B$ there exist a neighborhood $U_{\alpha^{0}}$ of $\alpha^{0}$ and a diffeomorphism

$$
h:\left\{(x, y) \mid f_{\alpha^{0}}(x, y)=0\right\} \times U_{\alpha^{0}} \rightarrow\left\{(x, y, \alpha) \mid f_{\alpha}(x, y)=0\right\} \cap \pi^{-1}\left(U_{\alpha^{0}}\right) .
$$

Consider the following two cases:

CASE 1: $0 \in C_{f}\left(f_{\alpha^{0}}\right)$. Since $h$ is a diffeomorphism of $\left\{(x, y) \mid f_{\alpha^{0}}(x, y)=0\right\}$ $\times U_{\alpha^{0}}$ onto $\left\{(x, y, \alpha) \mid f_{\alpha}(x, y)=0\right\} \cap \pi^{-1}\left(U_{\alpha^{0}}\right)$, it follows that $0 \in C_{f}\left(f_{\alpha}\right)$ for all $\alpha \in U_{\alpha^{0}}$, a contradiction.

CAse 2: $\alpha^{0} \in B_{\infty}$. In this case, the next lemma is needed.

2.4. LemMA. Let $F$ be a polynomial of two complex variables such that the restriction map $\left.l\right|_{V}, V:=F^{-1}(0)$, is proper where

$$
l: \mathbb{C}^{2} \rightarrow \mathbb{C}, \quad(x, y) \mapsto x .
$$

Suppose that the curve $V$ is reduced. Then

$$
\chi\left(F^{-1}(0)\right)=d-\operatorname{deg} \operatorname{disc}_{y} F(x, y) .
$$

Proof. Let $x_{\beta}, \beta=1, \ldots, p$, be the critical values of $\left.l\right|_{V}$ and $\left(x_{\beta}, y_{\beta}^{j}\right)$, $j=1, \ldots, i_{\beta}$, be the corresponding critical points of $\left.l\right|_{V}$ with multiplicity $l_{\beta}^{j}$. We may write

$$
\operatorname{disc}_{y} F(x, y)=a\left(x-x_{1}\right)^{\gamma_{1}} \cdots\left(x-x_{p}\right)^{\gamma_{p}},
$$

where $a \neq 0$ and $\gamma_{\beta}=\sum_{j=1}^{i_{\beta}} l_{\beta}^{j}, \beta=1, \ldots, p$.

Take $x_{*} \in \mathbb{C} \backslash\left\{x_{1}, \ldots, x_{p}\right\}$. Then $\left.l\right|_{V} ^{-1}\left(x_{*}\right)$ consists of $d:=\operatorname{deg}(F)$ distinct points. In the $x$-plane, we consider a system of paths $T_{1}, \ldots, T_{p}$ connecting $x_{1}, \ldots, x_{p}$ to $x_{*}$ such that

(i) no path $T_{j}$ has self-intersection points;

(ii) $T_{i} \cap T_{j}=\left\{x_{*}\right\}(i \neq j)$.

Put

$$
\widehat{\mathcal{S}}(V):=\left.l\right|_{V} ^{-1}\left(\bigcup_{i=1}^{p} T_{i}\right) .
$$


Then $\widehat{\mathcal{S}}(V)$ is a union of 1-dimensional curves. Let $\check{\mathcal{S}}(V)$ be the set of all curves in $\left.\widehat{\mathcal{S}}(V) \backslash l\right|_{V} ^{-1}\left(x_{*}\right)$ which contain a point of $\Sigma:=\left\{\left(x_{\beta}, y_{\beta}^{j}\right) \mid\right.$ $\left.j=1, \ldots, i_{\beta}, \beta=1, \ldots, p\right\}$.

Since $\bigcup_{i=1}^{p} T_{i}$ is a deformation retract of $\mathbb{C}$ and the restriction map

$$
\left.l\right|_{V}: F^{-1}(0) \backslash \Sigma \rightarrow \mathbb{C} \backslash\left\{x_{1}, \ldots, x_{p}\right\}, \quad(x, y) \mapsto x,
$$

is a locally trivial fibration, it follows that $\widehat{\mathcal{S}}(V)$ is a deformation retract of $V$.

It is not hard to see that

$$
\mathcal{S}(V):=\left.\check{\mathcal{S}}(V) \cup l\right|_{V} ^{-1}\left(x_{*}\right)
$$

is a deformation retract of $\widehat{\mathcal{S}}(V)$ and also of $V=F^{-1}(0)$. (The set $\mathcal{S}(V)$ is called the skeleton of the curve $V([2])$.) Hence,

$$
\chi\left(F^{-1}(0)\right)=\chi(\mathcal{S}(V)) .
$$

The set $\mathcal{S}(V)$ can be identified with a 1-dimensional graph of $d+\sum_{\beta=1}^{p} i_{\beta}$ vertices and $\sum_{\beta=1}^{p} \sum_{j=1}^{i_{\beta}}\left(l_{\beta}^{j}+1\right)$ edges. Thus,

$$
\begin{aligned}
\chi(\mathcal{S}(V)) & =\left(d+\sum_{\beta=1}^{p} i_{\beta}\right)-\sum_{\beta=1}^{p} \sum_{j=1}^{i_{\beta}}\left(l_{\beta}^{j}+1\right) \\
& =d-\sum_{\beta=1}^{p} \sum_{j=1}^{i_{\beta}} l_{\beta}^{j}=d-\sum_{\beta=1}^{p} \gamma_{\beta} \\
& =d-\operatorname{deg} \operatorname{disc}_{y} F(x, y) .
\end{aligned}
$$

We now return to Case 2 .

For each $\alpha \in \mathbb{C}^{n}$, let $k(\alpha):=\max \left\{j \in\{0, \ldots, k\} \mid q_{j}(\alpha) \neq 0\right\}$. By Lemma 2.4, we conclude that $\chi\left(f_{\alpha}^{-1}(0)\right)=d-k(\alpha)$. Since $\alpha^{0} \in B_{\infty}=q_{k}^{-1}(0)$ and $\alpha \notin B_{\infty}$ for generic $\alpha$, one gets

$$
\chi\left(f_{\alpha}^{-1}(0)\right)=d-k<\chi\left(f_{\alpha^{0}}^{-1}(0)\right)
$$

a contradiction, which ends the proof of Theorem 2.3.

2.5. Remark. From the construction of $B_{\infty}$, it is reasonable to call each $\alpha^{0} \in B_{\infty}$ a bifurcation value corresponding to the singularity at infinity of the family $\left\{f_{\alpha}(x, y)=0\right\}$.

3. Topology of families of affine plane curves. From now on, we assume that the curves $\left\{f_{\alpha}=0\right\}$ are smooth for generic $\alpha$.

Consider the family of affine curves $\left\{f_{\alpha}=0\right\}$. By Lemma 2.4, $\chi\left(f_{\alpha}^{-1}(0)\right)$ $=d-k(\alpha)$. On the other hand, by the definition of the set $B_{\infty}$, we see that $\alpha^{0} \in B_{\infty}$ iff $k\left(\alpha^{0}\right)<k=k(\alpha)$ for generic $\alpha$. Hence $\chi\left(f_{\alpha}^{-1}(0)\right)<\chi\left(f_{\alpha^{0}}^{-1}(0)\right)$ for generic $\alpha$. 
Thus we can reformulate Theorem 2.3 as follows.

3.1. Theorem. $\alpha^{0} \in \mathbb{C}^{n}$ is a bifurcation value of the family of affine curves $\left\{f_{\alpha}(x, y)=0\right\}$ if and only if either

(i) the curve $\left\{(x, y) \mid f_{\alpha^{0}}(x, y)=0\right\}$ is singular, or

(ii) $\chi\left(f_{\alpha}^{-1}(0)\right)<\chi\left(f_{\alpha^{0}}^{-1}(0)\right)$ for generic $\alpha$.

3.2. REMARK. In the general case, it is not sufficient to use the group $H_{1}\left(f_{\alpha}^{-1}(0)\right)$ to distinguish the generic curve from the special curve. For example, consider the family of polynomials $f_{\alpha}(x, y)=y^{3}+x y^{2}+y-\alpha$, $\alpha \in \mathbb{C}$. Then $B_{f}=\{0\}$ and $\operatorname{rank} H_{1}\left(f_{\alpha}^{-1}(0)\right)=\operatorname{rank} H_{1}\left(f_{0}^{-1}(0)\right)=1$.

There is no loss of generality in assuming that the map $l_{\alpha}=x$ is simple for each $\alpha$ near a given $\alpha^{0}$ ( $l_{\alpha}$ is said to be simple iff $l_{\alpha}^{-1}(x)$ consists of $d-1$ distinguished points for every critical value $x$ of $l_{\alpha}$ ). Then the number of singular points of $l_{\alpha}$ is exactly $k(\alpha)$. Let

$$
\left(x_{1}(\alpha), y_{1}(\alpha)\right), \ldots,\left(x_{k}(\alpha), y_{k}(\alpha)\right)
$$

be the critical points of the map $l_{\alpha}, \alpha \notin B_{\infty}$. Now we use the notations as in the proof of Lemma 2.4. Suppose that $x_{*} \in \mathbb{C}$ is a common regular value of $l_{\alpha^{0}}$ and $l_{\alpha}$ for all $\alpha$ near $\alpha^{0}$ and let $e_{j}(\alpha):=l_{\alpha}^{-1}\left(T_{j}\right) \cap S\left(f_{\alpha}^{-1}(0)\right), j=$ $1, \ldots, k$, be the cycles of the group $H_{1}\left(f_{\alpha}^{-1}(0), l_{\alpha}^{-1}\left(x_{*}\right)\right)$ corresponding to the singular points $\left(x_{j}(\alpha), y_{j}(\alpha)\right)$. These elements define a basis of the group $H_{1}\left(f_{\alpha}^{-1}(0), l_{\alpha}^{-1}\left(x_{*}\right)\right)$. By definition, $\alpha^{0} \in B_{\infty}$ iff there exist critical points $\left(x_{j}(\alpha), y_{j}(\alpha)\right)$ of $l_{\alpha}$ such that $\left\|\left(x_{j}(\alpha), y_{j}(\alpha)\right)\right\| \rightarrow \infty$ as $\alpha \rightarrow \alpha^{0}$. Moreover, since the map $l_{\alpha}$ is simple, the number of singular points of $l_{\alpha}$ tending to infinity as $\alpha \rightarrow \alpha^{0}$ is $r:=k-k\left(\alpha^{0}\right)$. Therefore, we may assume without loss of generality that such critical points are

$$
\left(x_{1}(\alpha), y_{1}(\alpha)\right), \ldots,\left(x_{r}(\alpha), y_{r}(\alpha)\right) .
$$

3.3. Definition. We call $e_{j}(\alpha), j=1, \ldots, r$, the semi-cycles vanishing at infinity as $\alpha \rightarrow \alpha^{0}$.

From Theorem 3.1 and the above definition we easily obtain the following.

3.4. TheOREM. $\alpha^{0}$ is a bifurcation value of the family of affine curves $\left\{f_{\alpha}(x, y)=0\right\}$ if and only if either

(i) the curve $\left\{(x, y) \mid f_{\alpha^{0}}(x, y)=0\right\}$ is singular, or

(ii) there exist semi-cycles $e_{j}(\alpha)$ vanishing at infinity as $\alpha \rightarrow \alpha^{0}$. The number of such semi-cycles is exactly $k-k\left(\alpha^{0}\right)$.

3.5. Remark. The number of semi-cycles $e_{j}(\alpha)$ vanishing at infinity can be given in other ways as follows. 
(i) Let $(F, G)$ be the intersection number of two curves $\{F=0\}$ and $\{G=0\}$. Then

$$
\begin{aligned}
\chi\left(f_{\alpha^{0}}^{-1}(0)\right)-\chi\left(f_{\alpha}^{-1}(0)\right)= & k-k\left(\alpha^{0}\right) \\
= & \left(f_{\alpha}, \partial f_{\alpha} / \partial y\right)-\left(f_{\alpha^{0}}, \partial f_{\alpha^{0}} / \partial y\right) \\
= & \operatorname{dim}_{\mathbb{C}} \mathbb{C}[x, y] /\left(f_{\alpha}, \partial f_{\alpha} / \partial y\right) \\
& -\operatorname{dim}_{\mathbb{C}} \mathbb{C}[x, y] /\left(f_{\alpha^{0}}, \partial f_{\alpha^{0}} / \partial y\right) .
\end{aligned}
$$

(ii) In $\mathbb{C P}^{2}$ we consider the family of curves

$$
\bar{\Gamma}_{\alpha}:=\left\{(x: y: z) \mid z^{d} f_{\alpha}(x / z, y / z)=0\right\} .
$$

Clearly, $\bar{\Gamma}_{\alpha}$ is the compactification of $\Gamma_{\alpha}:=f_{\alpha}^{-1}(0)$. In addition, we assume that the homogeneous part of degree $d$ of $f_{\alpha}$ does not depend on $\alpha$. Then the curves $\bar{\Gamma}_{\alpha}$ intersect the line $z=0$ at the same points $A_{1}, \ldots, A_{s}$ for any $\alpha$. Let $\mu_{A_{i}}\left(\bar{\Gamma}_{\alpha}\right)$ be the Milnor number of the germ of the analytic curve $\bar{\Gamma}_{\alpha}$ at $A_{i}$. By arguments of [3], we can show that

$$
\begin{aligned}
k-k\left(\alpha^{0}\right) & =\chi\left(f_{\alpha^{0}}^{-1}(0)\right)-\chi\left(f_{\alpha}^{-1}(0)\right) \\
& =\sum_{i=1}^{s}\left[\mu_{A_{i}}\left(\bar{\Gamma}_{\alpha^{0}}\right)-\mu_{A_{i}}\left(\bar{\Gamma}_{\alpha}\right)\right] .
\end{aligned}
$$

Next, we describe the change in the homotopy type of the curve $\left\{f_{\alpha}(x, y)=0\right\}$ as $\alpha \rightarrow \alpha^{0}, \alpha^{0} \in B_{\infty}$.

3.6. Definition. An operation of attaching a 1-dimensional cell to an affine curve $V$ is a map

such that

$$
\varphi: I:=[0,1] \rightarrow \mathbb{C}^{2}
$$

(i) $\varphi(I)$ is diffeomorphic to $I$;

(ii) $\varphi(I) \cap V=\{\varphi(0), \varphi(1)\}$.

The set $V^{\prime}=V \cup \varphi(I)$ is called $V$ with a 1-cell attached.

3.7. THEOREM. Let $\alpha^{0}$ be a bifurcation value corresponding to the singularity at infinity of the family $\left\{f_{\alpha}(x, y)=0\right\}$ such that the curve $\left\{f_{\alpha^{0}}(x, y)=0\right\}$ is smooth. Then a generic curve $f_{\alpha}^{-1}(0)$ may be obtained from $f_{\alpha^{0}}^{-1}(0)$, up to homotopy type, by attaching exactly $k-k\left(\alpha^{0}\right) 1$-dimensional cells.

Pr o of. Let $\mathcal{S}\left(f_{\alpha}^{-1}(0)\right)$ (resp. $\mathcal{S}\left(f_{\alpha^{0}}^{-1}(0)\right)$ ) be the skeleton of the affine plane curve $\left\{f_{\alpha}(x, y)=0\right\}$ (resp. $\left\{f_{\alpha^{0}}(x, y)=0\right\}$ ) as in the proof of Lemma 2.4. According to the construction of skeletons, the set $\mathcal{S}\left(f_{\alpha}^{-1}(0)\right)$ (resp. $\left.\mathcal{S}\left(f_{\alpha^{0}}^{-1}(0)\right)\right)$ is a graph with $d+k\left(\right.$ resp. $\left.d+k\left(\alpha^{0}\right)\right)$ vertices and $2 k$ (resp. $\left.2 k\left(\alpha^{0}\right)\right)$ edges. 
Furthermore, $\mathcal{S}\left(f_{\alpha^{0}}^{-1}(0)\right)$ is obtained from $\mathcal{S}\left(f_{\alpha}^{-1}(0)\right)$ by deleting $k-k\left(\alpha^{0}\right)$ vertices $\left(x_{\beta}(\alpha), y_{\beta}(\alpha)\right), \beta=1, \ldots, r$, and $k-k\left(\alpha^{0}\right)$ pairs of edges. These pairs of edges connect a point $\left(x_{\beta}(\alpha), y_{\beta}(\alpha)\right)$ tending to infinity to two distinct points in $l_{\alpha}^{-1}\left(x_{*}\right)$. In other words, the set $\mathcal{S}\left(f_{\alpha}^{-1}(0)\right)$ is $\mathcal{S}\left(f_{\alpha^{0}}^{-1}(0)\right)$ with $k-$ $k\left(\alpha^{0}\right)$ 1-cells attached.

On the other hand, the graph $\mathcal{S}\left(f_{\alpha}^{-1}(0)\right)$ (resp. $\left.\mathcal{S}\left(f_{\alpha_{0}}^{-1}(0)\right)\right)$ is a deformation retract of the curve $f_{\alpha}^{-1}(0)$ (resp. $f_{\alpha_{0}}^{-1}(0)$ ). This proves the theorem.

3.8. COROllary. If $\alpha^{0}$ is a bifurcation value at infinity of the family of curves $\left\{f_{\alpha}=0\right\}$, then the number of connected components of the curve $f_{\alpha^{0}}^{-1}(0)$ is greater than or equal to the one for $f_{\alpha}^{-1}(0)$.

Moreover, we can describe a change mechanism of the number of connected components when passing from the general curves $f_{\alpha}^{-1}(0)$ to the special curve $f_{\alpha^{0}}^{-1}(0)$. For that, we need:

3.9. Definition. A subgraph $B(\alpha)$ of the graph $S\left(f_{\alpha}^{-1}(0)\right)$ is said to be a block vanishing at infinity as $\alpha \rightarrow \alpha^{0}$ if the following four conditions are satisfied.

(i) $B(\alpha)$ is connected;

(ii) each vertex of $B(\alpha)$ either belongs to $l_{\alpha}^{-1}\left(x_{*}\right)$ or tends to infinity as $\alpha \rightarrow \alpha^{0}$

(iii) the number of connected components of $S\left(f_{\alpha}^{-1}(0)\right)$ is different from that of $S\left(f_{\alpha}^{-1}(0)\right) \backslash B(\alpha)$;

(iv) $B(\alpha)$ is minimal in the sense that there exists no subgraph $B^{\prime}(\alpha) \nsupseteq$ $B(\alpha)$ of $S\left(f_{\alpha}^{-1}(0)\right)$ satisfying (i)-(iii).

Let $v\left(\alpha^{0}\right)$ be the number of blocks vanishing at infinity as $\alpha \rightarrow \alpha^{0}$, and let $b_{0}(\alpha)$ and $b_{0}\left(\alpha^{0}\right)$ be the numbers of connected components of $f_{\alpha}^{-1}(0)$ and $f_{\alpha^{0}}^{-1}(0)$, respectively. By Theorem 3.7, we obtain the following.

3.10. Theorem. $b_{0}\left(\alpha^{0}\right)-b_{0}(\alpha)=v\left(\alpha^{0}\right)$.

\section{Corollaries}

4.1. We begin by recalling some facts on the topology of polynomials of two variables.

Let $F: \mathbb{C}^{2} \rightarrow \mathbb{C}$ be a polynomial function. It is well known that there exists a finite set $C(F) \subset \mathbb{C}$, called the bifurcation set of $F$, such that the restriction

$$
F: \mathbb{C}^{2} \backslash F^{-1}(C(F)) \rightarrow \mathbb{C} \backslash C(F)
$$

is a locally trivial $C^{\infty}$-fibration (see, for example, [5], [6], [7], [3]).

We say that a value $t_{0} \in \mathbb{C}$ is regular at infinity if there exist a small $\delta>0$ and a compact $K \subset \mathbb{C}^{2}$ such that the restriction 


$$
F: F^{-1}\left(D_{\delta}\right) \backslash K \rightarrow D_{\delta}, \quad D_{\delta}:=\left\{t|| t-t_{0} \mid<\delta\right\},
$$

is a trivial $C^{\infty}$-fibration ([4]).

If $t_{0}$ is not regular at infinity, it is called a critical value at infinity of $F$. Denote by $C_{\infty}(F)$ the set of critical values at infinity of $F$. It is known ([3]) that $C(F)=C_{f}(F) \cup C_{\infty}(F)$.

Let $F$ be a polynomial with isolated critical points only. Denote by $\mu^{c}(F)$ the fibre Milnor number of $F$ at $c$.

Let $f_{\alpha}(x, y):=F(x, y)-\alpha, \alpha \in \mathbb{C}$. Using the notation of Remark 3.5, we put

$$
\lambda^{\alpha^{0}}(F):=\sum_{i=1}^{s}\left[\mu_{A_{i}}\left(\bar{\Gamma}_{\alpha}\right)-\mu_{A_{i}}\left(\bar{\Gamma}_{\alpha^{0}}\right)\right],
$$

for $\alpha^{0} \in \mathbb{C}$ and generic $\alpha$.

Now, let us mention an important consequence of the above results.

4.2. Corollary. The following statements are equivalent.

(i) $\alpha^{0} \in B_{f}$;

(ii) $0 \in C\left(f_{\alpha^{0}}\right)$;

(iii) $\mu^{0}\left(f_{\alpha^{0}}\right)+\lambda^{0}\left(f_{\alpha^{0}}\right)>0$.

Proof. We first show that

$$
B_{\infty}=\left\{\alpha \mid 0 \in C_{\infty}\left(f_{\alpha}\right)\right\} .
$$

In fact, let $\Delta(x, \alpha, t):=\operatorname{disc}_{y}\left(f_{\alpha}(x, y)-t\right)$. Then we may write

$$
\Delta(x, \alpha, t)=Q_{m(\alpha)}(\alpha, t) x^{m(\alpha)}+Q_{m(\alpha)-1}(\alpha, t) x^{m(\alpha)-1}+\ldots
$$

According to [1],

$$
C_{\infty}\left(f_{\alpha}\right)=\left\{t \in \mathbb{C} \mid Q_{m(\alpha)}(\alpha, t)=0\right\} .
$$

On the other hand, since $0 \notin C_{\infty}\left(f_{\alpha}\right)$ for generic $\alpha$,

$$
Q_{m(\alpha)}(\alpha, 0) \not \equiv 0 \text {. }
$$

Furthermore, because $\delta(x, \alpha)=\Delta(x, \alpha, 0)$, we have

$$
Q_{m(\alpha)}(\alpha, 0) \equiv q_{k}(\alpha) \text { and } m(\alpha) \equiv k .
$$

Therefore,

$$
B_{\infty}=\left\{\alpha \mid q_{k}(\alpha)=0\right\}=\left\{\alpha \mid Q_{m(\alpha)}(\alpha, 0)=0\right\}=\left\{\alpha \mid 0 \in C_{\infty}\left(f_{\alpha}\right)\right\} .
$$

We now prove the theorem. By [1], $t^{0} \in C_{\infty}\left(f_{\alpha}\right)$ iff

$$
\lambda^{t^{0}}\left(f_{\alpha}\right)>0 \text {. }
$$

By (9), (10) and the definition of $B_{f}$, one has $B_{f}=\left\{\alpha \mid 0 \in C\left(f_{\alpha}\right)\right\}$, from which the assertion easily follows.

In a special case, the following corollary is well known. 
4.3. Corollary $([3],[6])$. Suppose that $F \in \mathbb{C}[x, y]$ is a polynomial of two complex variables. Then $\alpha^{0} \in C(F)$ if and only if either

(i) $\alpha^{0}$ is a singular value of $F$, or

(ii) $\chi\left(F^{-1}(\alpha)\right)<\chi\left(F^{-1}\left(\alpha^{0}\right)\right)$ for generic $\alpha$.

Proof. Let $f_{\alpha}(x, y):=F(x, y)-\alpha, \alpha \in \mathbb{C}$. Then the conclusion follows from Theorem 3.1 and Corollary 4.2.

4.4. Remark. Let $f_{\alpha}(x, y)=F(x, y)-\alpha$. Then the results of $\S 3$ also give us the corresponding results of [2] on the semi-cycles vanishing at infinity and on the construction of the homotopy type of the generic fiber for a global Milnor fibration.

\section{References}

[1] Hà Huy Vui, Sur la fibration globale des polynômes de deux variables complexes, C. R. Acad. Sci. Paris Sér. I 309 (1989), 231-234.

[2] —, La formule de Picard-Lefschetz affine, ibid. 321 (1995), 747-750.

[3] Hà Huy Vui et Lê Dũng Tráng, Sur la topologie des polynômes complexes, Acta Math. Vietnam. 9 (1984), 21-32.

[4] W. D. Neumann, Complex algebraic plane curves via their links at infinity, Invent. Math. 98 (1989), 445-489.

[5] F. Pham, Vanishing homologies and the $n$ variables saddlepoint method, in: Proc. Sympos. Pure Math. 40, Amer. Math. Soc., 1983, 310-333.

[6] M. Suzuki, Propriétés topologiques des polynômes de deux variables complexes, et automorphismes algébriques de l'espace $\mathbb{C}^{2}$, J. Math. Soc. Japan 26 (1974), 241-257.

[7] R. Thom, Ensembles et morphismes stratifiés, Bull. Amer. Math. Soc. 75 (1969), 249-312.

Institute of Mathematics

Department of Mathematics

P.O. Box 631

Bo-Ho, Hanoi, Vietnam

Dalat University

E-mail: hhvui@ioit.ncst.ac.vn

Dalat, Vietnam 